\title{
Editorial
}

\section{HIV Surveillance: The Conscience of the Epidemic}

\author{
Patrick S. Sullivan ${ }^{*}, 1$ and Valerie Delpech ${ }^{2}$ \\ ${ }^{I}$ Department of Epidemiology, Rollins School of Public Health, 1518 Clifton Road NE, Atlanta, GA 30322, USA \\ ${ }^{2}$ Health Protection Agency, HIV \& STI Department - HIV and AIDS Reporting Section, UK
}

The first cases of what would come to be recognized as the beginning of the HIV epidemic were reported without a lot of fanfare in the US CDC's Morbidity and Mortality Weekly Report [1]. The report of five homosexual men with a rare pneumonia was presented as a case series, but, in retrospect, established a working surveillance case definition for a new disease. Dr. James Curran, the scientist who led the CDC team for that MMWR report, later oversaw the US HIV surveillance system for many years and has famously called HIV surveillance "the conscience of the epidemic".

Dr. Curran's statement (like all good truisms), works on many levels. In its simple context, HIV surveillance data on counts of cases have been objective measures of our challenges and our successes in responding to the epidemics in many countries throughout the world. Surveillance data, objectively presented and interpreted, should be used as guideposts for determining how prevention resources can be best allocated, and for planning what resources will be required for those living with HIV. On another level, our current understanding of surveillance data describing the extent of late diagnoses and health disparities by gender, poverty, and race in HIV epidemics speak to the obligations of our communities to address issues of prompt access to HIV testing, treatment and care, and attention to the socioeconomic factors that are critical drivers of many HIV epidemics.

Through the decades, HIV surveillance systems have informed our increasingly detailed understanding of the disease in populations, and advances in understanding of the transmission dynamics of the infection and its management have driven refinements in the surveillance systems [2]. As public health activity evolves--to embrace new technologies, to address new challenges (e.g., resistant HIV), to investigate new approaches to old challenges (e.g., health disparities), to expand into new ways of discharging essential functions (i.e., assuring linkage and retention and the quality of HIV care) - the surveillance foundation supporting it has to evolve as well. Surveillance approaches have arisen that are suited to the epidemiologic conditions, public health infrastructures, and resources available in diverse regions of the world. In some countries, especially those with generalized

*Address correspondence to this author at the Department of Epidemiology, Rollins School of Public Health, 1518 Clifton Road NE, Atlanta, GA 30322, USA; Tel: 404-727-2038; E-mail: pssulli@emory.edu epidemics, public health surveillance has integrated with broader, demographic and health surveys. The span of surveillance activities has grown in many countries to include the surveillance of behaviors that are antecedent to HIV infection and co-infections, broadening the potential for public health monitoring and intervention at points before infection occurs [3]. Clinical outcomes surveillance approaches produce high quality data to describe quality of care, and to document the impact of HIV care and treatment funding on reducing morbidity and mortality [4]. Surveillance data serve as a crucial benchmark against which other data and research cohorts can be measured to understand representativeness and biases [5].

The manuscripts in this special issue represent the innovation of HIV surveillance around the world. New methods, such as probability sampling for clinical outcomes surveillance and methods to develop population estimates for most at risk subpopulations, improve the representativeness of data [6]. Use of surveillance data for evaluation, such as repeat western blot testing in South Carolina [7] and CD4 reporting in the United Kingdom [8], illustrate how surveillance data can be used to increase efficiency in use of care resources and public reporting efforts. Analyses of clinical outcomes surveillance data, such as the description of coronary heart disease [9], can help describe emerging threats to the health of people living with HIV and changing health circumstances as infected populations age. Internetbased approaches allow public health scientists to reach populations for behavioral surveillance in new venues of risk, and with increased resource efficiency [10].

Several papers in this issue highlight the methods underlying two of CDC's supplemental surveillance systems: the National HIV Behavioral Surveillance System (NHBS) and the Medical Monitoring Project (MMP). The use of innovative and complex sampling methods is described for NHBS among men who have sex with men (using the internet [11]) injection drug users [12] and heterosexuals at risk [13], as well as for MMP to reach a representative sample of persons in care in the United States [6]. The program of HIV surveillance activities in the United States, supported by the US CDC, provides for data along a spectrum of surveillance from risk to diagnosis to care and treatment. This unique combination of data allows for more detailed description of the burden of HIV, monitoring progress via key indicators used to benchmark national prevention goals, and evaluate outcomes and impacts of 
prevention. Putting these articles together in a special issue underscores the breadth of information collected through these systems and brings to light the challenges of designing and implementing innovative, complex surveillance systems.

Another critical message about HIV surveillance that emerges from the manuscripts collected here is the imperative to disseminate surveillance data at all levels in the surveillance enterprise. The assembled reports include analyses from the city, state, and national levels of surveillance systems, and from three continents. Surveillance uses the power of the state to compel the collection of limited information for the common purpose of improving and monitoring public health response, and those who collect and hold the data have an obligation to disseminate it in ways that improve transparency, and improve community response. Disseminating these findings in an open access format is especially important, because surveillance data should be democratic, and many potential users and stakeholders, especially those within low and middle income countries, do not have access to institutional subscriptions or resources to pay per-article usage fees.

In the fourth decade, HIV surveillance should continue to be the conscience of the epidemic. The works of the authors presented in this special issue, and of the institutions and stakeholders that they represent, exemplify the highest standard of public health practice. We hope that these manuscripts will motivate you to strive for new and reinvigorated responses to the HIV epidemic in your own community.

\section{REFERENCES}

[1] Centers for Disease Control and P. Pneumocystis Pneumonia --Los Angeles. Morb Mortal Wkly Rep 1981; 30(21): 1-3.

[2] Nakashima AK, Fleming PL. HIV/AIDS surveillance in the United States, 1981-2001. J Acquir Immune Defic Syndr 2003; 32(Suppl 1): S68-S85.
[3] Gallagher K, Sullivan PS, Lansky A. Behavioral surveillance among persons at risk for HIV infection in the US: The National HIV Behavioral Surveillance System. Public Health Rep 2007; 122(Suppl 1): 32-38.

[4] McNaghten AD, Wolfe MI, Onorato IM, et al. Improving behavioral and clinical HIV/AIDS surveillance in the United States: the rationale for developing a population-based approach. PLoS One 2007; 2(6): e550.

[5] Donnell D. Use Of HIV Case Surveillance System To Design And Evaluate Site-Randomized Interventions In An HIV Prevention Study: HPTN 065 (TLC-Plus Study). Open AIDS J 2012; 6: 000000 .

[6] Frankel MR. A Probability Sample for Monitoring the HIVinfected Population in Care in the U.S. and in Selected States. Open AIDS J 2012; 6: 000-000.

[7] Duffus W. Repeat Western Blot Testing after Receiving an HIV Diagnosis and Its Association with Engagement in Care. Open AIDS J 2012; 6: 000-000.

[8] Brown AE. Auditing national HIV guidelines and policies: the UK CD4 surveillance scheme. Open AIDS J 2012; 6: 000-000.

[9] Oh MK. Population-Based Study of Risk Factors for Coronary Heart Disease Among HIV-Infected Persons. Open AIDS J 2012; 6: 000-000.

[10] Sanchez T. Developing a Web-based HIV Behavioral Surveillance Pilot Project among Men Who Have Sex with Men. Open AIDS J 2012; 6: 000-000.

[11] Sanchez T. Internet-based Methods May Reach Higher-Risk Men who have Sex with Men Not Reached through Venue-based Sampling. Open AIDS J 2012; 6: 000-000.

[12] Lansky A. Assessing the Assumptions of Respondent-Driven Sampling in the National HIV Behavioral Surveillance System among Injecting Drug Users. Open AIDS J 2012; 6: 000-000.

[13] Dinenno E. Piloting a System for Behavioral Surveillance among Heterosexuals at Increased Risk of HIV in the United States. Open AIDS J 2012; 6: 000-000. 